\title{
OPEN Analysis of extracellular vesicle mRNA derived from plasma using the nCounter platform
}

\author{
Jillian W. P. Bracht ${ }^{1,2 \bowtie}$, Ana Gimenez-Capitan ${ }^{1}$, Chung-Ying Huang ${ }^{3}$, Nicolas Potie ${ }^{4,5}$, \\ Carlos Pedraz-Valdunciel ${ }^{2,6}$, Sarah Warren ${ }^{3}$, Rafael Rosell ${ }^{6}$ \& Miguel A. Molina-Vila ${ }^{16}$
}

Extracellular vesicles (EVs) are double-layered phospholipid membrane vesicles that are released by most cells and can mediate intercellular communication through their RNA cargo. In this study, we tested if the NanoString nCounter platform can be used for the analysis of EV-mRNA. We developed and optimized a methodology for EV enrichment, EV-RNA extraction and nCounter analysis. Then, we demonstrated the validity of our workflow by analyzing EV-RNA profiles from the plasma of 19 cancer patients and 10 controls and developing a gene signature to differentiate cancer versus control samples. TRI reagent outperformed automated RNA extraction and, although lower plasma input is feasible, $500 \mu \mathrm{L}$ provided highest total counts and number of transcripts detected. A 10-cycle preamplification followed by DNase treatment yielded reproducible mRNA target detection. However, appropriate probe design to prevent genomic DNA binding is preferred. A gene signature, created using a bioinformatic algorithm, was able to distinguish between control and cancer EV-mRNA profiles with an area under the ROC curve of 0.99 . Hence, the nCounter platform can be used to detect mRNA targets and develop gene signatures from plasma-derived EVs.

With the growing global cancer burden, estimated at 18 million cases in $2018^{1}$, earlier cancer detection, enhanced disease monitoring and improved therapy selection are indispensable to enhance patient survival. Liquid biopsies provide a minimally invasive, safe and sensitive surrogate for tissue biopsies. Extracellular vesicles (EVs) are double-layered phospholipid membrane vesicles that are released by most cells, including cancer cells, immune cells and even blood platelets, and can be isolated from practically any body fluid. Cells liberate highly heterogeneous EVs in terms of size $(10 \mathrm{~nm}-1 \mu \mathrm{m})$, cargo (nucleic acids, proteins and lipids), membrane composition, biogenesis and biological function ${ }^{2-4}$. Several EV enrichment strategies have been described, including ultracentrifugation, size-exclusion chromatography and precipitation. Precipitation buffers capture water molecules and thereby decrease the hydration of particles, allowing their precipitation after a low-speed centrifugation.

Importantly, the active molecules that are found within EVs can be transported to local or distant target cells and execute biological functions, making EVs important mediators of intercellular communication ${ }^{2,5,6}$. Since the quantity of released EVs and their specific cargo is regulated by the producing cells, the RNA profiles contained within EVs could potentially be used as biomarkers for development and progression of several diseases, including cancer ${ }^{7}$. EVs also have the advantage of their lipid bilayer, which makes their cargo particularly stable and allows the use of biobank stored samples.

Gene expression studies using EV-RNA from cancer patients are an active area of research ${ }^{8,9}$. Promising findings have been reported but the lack of standardized protocols for RNA extraction and analysis, leading to inconsistent results, is hampering clinical implementation. Transcriptomic analysis studies are often conducted using quantitative real time PCR (qRT-PCR), microarrays or RNA sequencing (RNAseq), each with their own (dis)advantages ${ }^{10}$. While qRT-PCR provides high sensitivity and specificity with a short turnaround time, it only allows for low-throughput transcriptomic analysis of a limited number of genes. Microarrays represent a medium-throughput platform but have a narrower dynamic range of detection and are not suitable to detect

\footnotetext{
${ }^{1}$ Pangaea Oncology, Laboratory of Oncology, Quirón Dexeus University Hospital, Sabino Arana 5-19, 08028 Barcelona, Spain. ${ }^{2}$ Department of Biochemistry, Molecular Biology and Biomedicine, Universitat Autónoma de Barcelona (UAB), 08193 Cerdanyola, Spain. ${ }^{3}$ NanoString Technologies, Seattle, WA, USA. ${ }^{4}$ Department of Genetics, Faculty of Science, University of Granada, 18071 Granada, Spain. ${ }^{5}$ Bioinformatics Laboratory, Biotechnology Institute, Centro de Investigacion Biomedica, PTS, Avda. del Conocimiento s/n, 18100 Granada, Spain. ${ }^{6}$ Germans Trias i Pujol Health Sciences Institute and Hospital (IGTP), Badalona, Barcelona, Spain. ${ }^{\boxplus}$ email: jill94bracht@gmail.com; mamolina@panoncology.com
} 
A

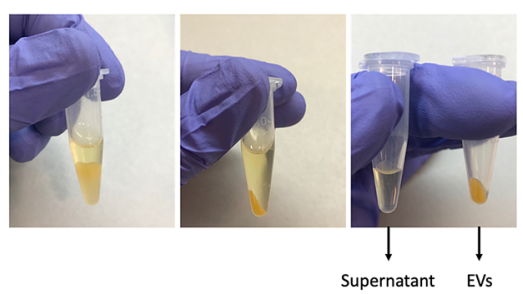

c

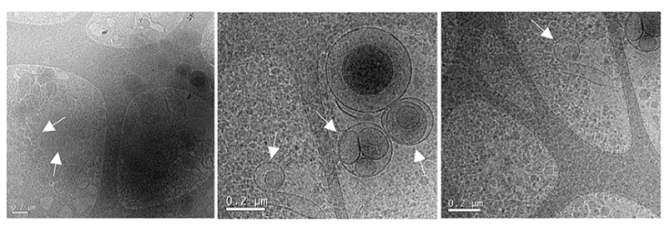

$\mathrm{E}$
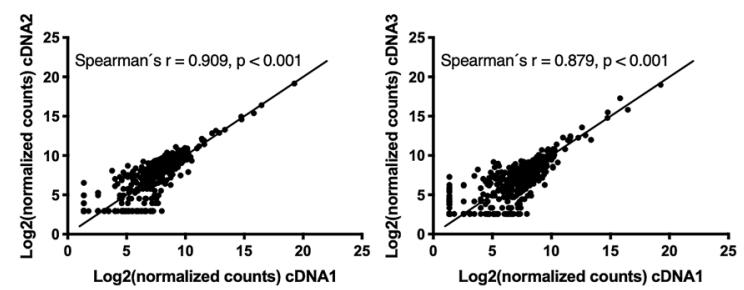

B

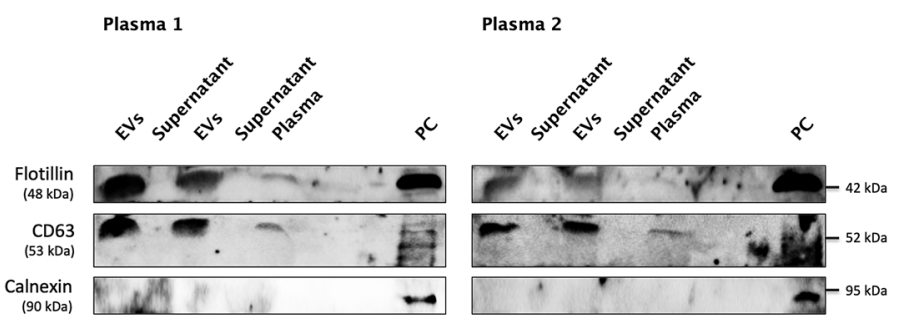

D
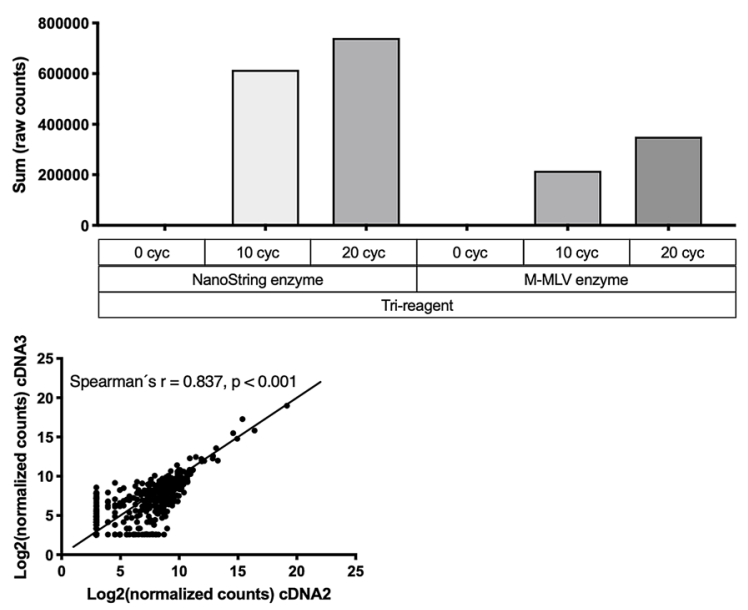

Figure 1. EV enrichment and characterization, assay reproducibility. (A) EV enrichment based on precipitation using the miRCURY Exosome Serum/Plasma Kit, including separation of supernatant and EV-enriched pellet. (B) Immunoblots showing expression of Flotillin, CD63 (both exosome markers) and Calnexin (cell specific marker) in EV-enriched pellets, supernatants, full plasma and a positive control sample. Experiments were performed in duplicates. Membranes were cut and incubated with specific antibodies for Flotillin, CD63 and Calnexin. Images were cropped for clarity purposes and full membranes can be found in Supplementary Fig. S1. (C) Cryogenic Electron Microscopy (cryo-EM) of EV pellets. Arrows point to extracellular vesicles with different size ranges. Scale bars are $200 \mathrm{~nm}$. (D) Total counts by nCounter after EV-RNA extraction using TRI reagent. Two different retrotranscriptases (NanoString versus M-MLV) and three pre-amplification conditions $(0,10$ and 20 cycles) were tested. (E) Reproducibility experiment comparing the Log2 normalized counts by nCounter from three independent cDNAs derived from a single EV-RNA sample. Spearman's correlation coefficient is indicated. EVs, extracellular vesicles; PC, positive control; Cyc, cycles.

genes expressed at either low or high levels. Finally, RNAseq is an accurate, high-throughput platform with a wide dynamic range, but limitations include longer turnaround time, high cost and complex data analysis ${ }^{11,12}$.

In recent years, the NanoString nCounter platform has gained popularity in translational research and clinical settings. The platform provides a simple, sensitive and cost-effective solution for multiplexed analysis of up to 800 RNA targets by direct capturing and counting of individual targets. In addition, it can be used with formalin-fixed paraffin embedded (FFPE) tumor tissue and allows for low quality and quantity tissue samples ${ }^{13,14}$. At this respect, the nCounter-based Prosigna assay, which differentiates breast cancer subtypes and predicts the risk of recurrence based on a 50-gene signature, has been validated in the clinical practice and received FDA approval in $2013^{15,16}$. Another assay developed using the nCounter platform is the 18-gene Tumor Inflammation Signature (TIS), which was able to predict clinical response to PD-1 blockade in an investigational clinical trial assay ${ }^{17}$. These two assays, which utilize tissue samples, emphasize the potential of the nCounter platform as biomarker assay development tool, especially in diagnostic laboratories. Regarding the analysis of liquid biopsies on nCounter, several studies have investigated the potential of some materials, including $\mathrm{cf}^{-18}$ and EV-DNA ${ }^{19}$, CTC-RNA ${ }^{20,21}$, leukocyte mRNA ${ }^{22}$, cfRNA ${ }^{23}$ and EV-miRNA ${ }^{24,25}$, with different success rates. However, nCounter has never been tested for the analysis of EV-derived mRNA.

Here, we present a proof-of-concept study where we optimized a workflow for EV enrichment from human blood samples, EV-mRNA purification and subsequent analysis by nCounter. Then, we used the workflow to develop an EV-mRNA based gene signature to differentiate cancer versus control samples. Our work demonstrates that nCounter can be employed for biomarker discovery based on EV-mRNA.

\section{Results}

Optimization of plasma EV enrichment and EV-RNA extraction methodologies. EVs were enriched from $500 \mu \mathrm{L}$ plasma of control samples using the miRCURY Exosome Serum/Plasma Kit (Fig. 1a). Final miRCURY sediments were submitted to western blotting, revealing enrichment in the exosome markers Flotillin and CD63, which were absent or detected at low levels in miRCURY supernatants and whole plasma samples. 
A

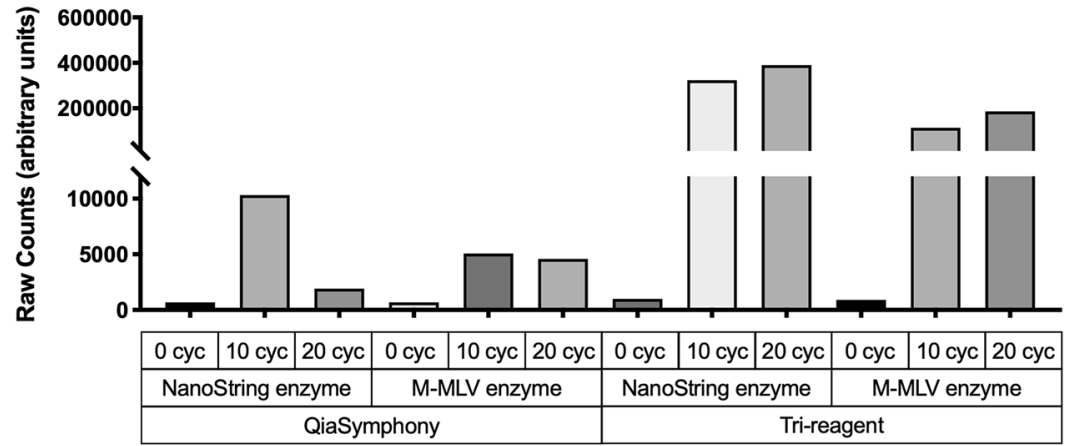

C

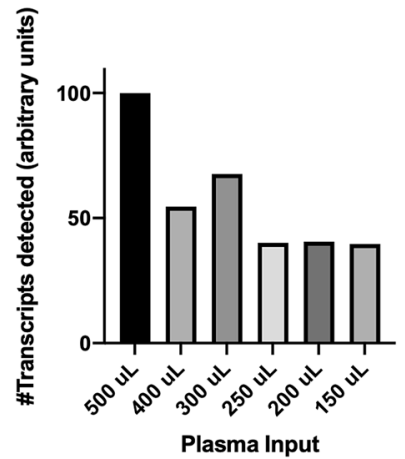

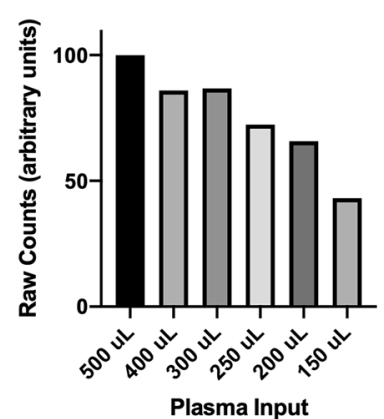

B

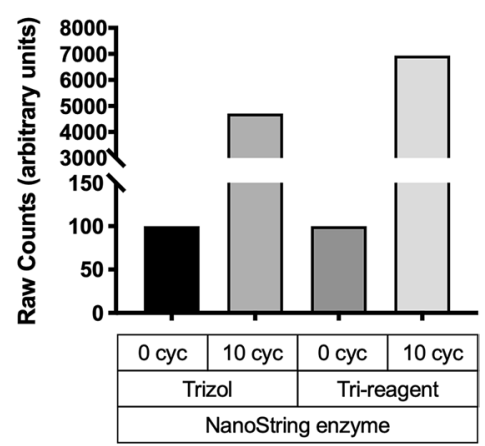

D

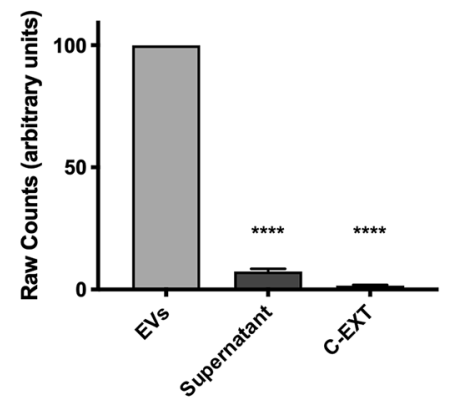

Figure 2. EV-RNA extraction, targeted pre-amplification and plasma input testing. (A) Total nCounter counts after automated (QiaSymphony) versus manual (TRI reagent) RNA extraction from an EV-enriched pellet. Two different retrotranscriptases (NanoString versus M-MLV) and three pre-amplification conditions (0, 10 and 20 cycles) were tested. Results were normalized to the counts corresponding to 0 cycles. (B) Total nCounter counts after TRIzol LS versus TRI reagent manual RNA isolation from an EV-enriched pellet. Results were normalized to the counts corresponding to 0 cycles. (C) Effect of input plasma volume (150-500 $\mu \mathrm{L}$ ) on the final number of transcripts detected (left) or total nCounter counts (right) Results were normalized to the counts corresponding to $500 \mu \mathrm{L}$ plasma. (D) Total nCounter counts of different fractions obtained during EV enrichment of plasma (EVs vs. supernatant $p<0.0001$ in a one-way ANOVA with Dunnett's multiple comparisons test; EVs versus C-EXT $p<0.0001)$. Cyc, cycles; EVs, extracellular vesicles; C-EXT, extraction control.

Sediments, supernatants and plasmas were negative for the cell-specific marker calnexin (Fig. 1b, Supplementary Fig. S1). Cryogenic electron microscopy (cryo-EM), a commonly used technique for EV characterization ${ }^{26,27}$, was used to visualize the miRCURY sediments, revealing EVs with the classical morphology and a diameter of $100-300 \mathrm{~nm}$, in agreement with the reported $10-1 \mu \mathrm{m}$ size range (Fig. 1c) $)^{2-4}$.

TRIzol LS and TRI reagent are mixtures of phenol, guanidine isothiocyanate and other components routinely used for nucleic acid extractions. We found that the quantity of RNA that could be isolated from EV-enriched sediments using TRI reagent was too low to be determined by the Qubit RNA High Sensitivity Assay Kit (Thermo Fisher Scientific). Bioanalyzer profiles of two representative samples revealed RNA concentrations $<150 \mathrm{pg} /$ $\mu \mathrm{L}$, insufficient for nCounter analysis using the Human Immunology V2 Panel, which we had selected for our study (Supplementary Fig. S2). Therefore, we tested retrotranscription and pre-amplification of the EV-mRNA with the nCounter Low RNA Input Amplification Kit, using primers targeting the genes of the Panel. Two reverse transcriptases were compared, the M-MLV and the enzyme provided by the kit, together with 10 versus 20 -cycles for the pre-amplification step. Results indicated that the retrotranscriptase provided by the kit was more efficient in terms of final counts and that both 10 and 20 cycles yielded sufficient raw counts for successful nCounter analysis with the Human Immunology V2 Panel (Fig. 1d). However, 20-cycles of pre-amplification led to saturation for some genes with higher expression levels (Supplementary Fig. S2). In consequence, 10 cycles were selected for the final workflow.

To test the reproducibility of the steps described above, we retrotranscribed and pre-amplified the same EV-RNA sample on three independent reactions. Then, we compared the results obtained when submitting the three resulting cDNA samples to nCounter analysis. A strong correlation was found between the normalized counts for each individual gene obtained in the different cDNAs, represented by a Spearman's $r$ of $0.84-0.91$, $p<0.01$ (Fig. 1e).

All the experiments so far described had been performed with $500 \mu \mathrm{L}$ plasma samples and TRI reagent. Two additional RNA extraction methods were tested on EV-enriched preparations from control samples, the automated QiaSymphony and the manual TRIzol LS Reagent based isolation (Fig. 2a). When considering the total number of counts by nCounter, TRI reagent was found to outperform both QiaSymphony and TRIzol LS, independently of the retrotranscriptase or the number of cycles used for the pre-amplification step (Fig. 2b). Finally, we also tested the effect of plasma input volume on downstream analysis of EV-RNA on the nCounter platform. Both the total counts and number of transcripts detected were higher with an initial plasma volume of $500 \mu \mathrm{L}$ (Fig. 2c). 


\begin{tabular}{|l|l|l|}
\hline Characteristics & Cancer patients (n=19) & Controls (n=10) \\
\hline Gender-no. (\%) & $11(57.9)$ & $5(50.0)$ \\
\hline Male & $8(42.1)$ & $5(50.0)$ \\
\hline Female & \multicolumn{2}{|l|}{} \\
\hline Age-year & 62 & 42 \\
\hline Median & $45-78$ & $24-53$ \\
\hline Range & $10(52.7)$ & - \\
\hline Tumor type-no. (\%) & \multicolumn{2}{|l|}{} \\
\hline Lung (ADC) & $3(15.8)$ & - \\
\hline Gastric (ADC) & $2(10.5)$ & - \\
\hline Anal (SCC) & $2(10.5)$ & - \\
\hline Rectal (ADC) & $2(10.5)$ & - \\
\hline Sarcoma (UPS) & & - \\
\hline Stage-no. (\%) & $2(10.5)$ & - \\
\hline Stage III & $17(89.5)$ & - \\
\hline Stage IV & &
\end{tabular}

Table 1. Clinical characteristics of the cancer patients and controls included in the study. ADC, adenocarcinoma; SCC, squamous cell carcinoma; UPS, undifferentiated pleomorphic sarcoma.

RNase $\mathrm{A}$ is a bovine enzyme that can be used to degrade RNA in a sample. When enriching for EVs from plasma samples, we expect two sources of RNA; extra-vesicular RNA and RNA derived from within the EVs. Our workflow for EV-RNA analysis incorporates an RNase A treatment of the EV-enriched pellets in order to remove extra-vesicular RNA not embedded into the particles. To further validate this step, we collected two EV-enriched pellets and the corresponding supernatants of healthy patients, treated them with RNase A, purified the RNA and analyzed them with the Human Immunology V2 Panel. We found that EV-enriched pellets yielded significantly higher transcript counts compared to the supernatant (one-way ANOVA with Dunnett's multiple comparisons, EVs versus supernatant $p<0.01$ ), indicating that the transcripts detected by nCounter are associated with EVs (Fig. 2d). To validate the efficacy of our RNase treatment, we also checked the expression of GAPDH and CCL5 by qRT-PCR in three patient samples. Three different sample conditions were used; EVs without RNase treatment, EVs with RNase treatment and lysed EVs with RNase treatment. Results indicated that RNase treatment of intact EVs slightly reduced the GAPDH and CCL5 mRNA levels, probably by removing the extra-vesicular RNA co-precipitated with the EVs (Supplementary Fig. S3). In contrast, once EVs were lysed, RNase treatment effectively eliminated all RNA and both GAPDH and CCL5 transcripts were undetectable, confirming that transcripts purified using our workflow are indeed contained within the EVs.

EV-derived mRNA analysis on nCounter and classifier development. Based on the data presented above, we selected a workflow for subsequent experiments; starting with EV enrichment from $500 \mu \mathrm{L}$ of plasma with the miRCURY kit, followed by manual RNA purification with TRI reagent, retrotranscription with the Nanostring enzyme, a 10-cycle pre-amplification and final cDNA analysis by nCounter. We validated the proposed workflow by studying the EV-mRNA expression of 19 cancer patient and 10 control plasma samples using the Human Immunology V2 Panel (Table 1). If all samples were considered together, the average number of transcripts detected was $430 \pm 79$ out of the 594 transcripts in the panel (Fig. 3a). No significant differences were found between the number of mRNA transcripts in EVs from controls versus cancer patients ( $445 \pm 68$ and $422 \pm 84$ respectively, Mann-Whitney's U $p=0.46$ ).

After normalization, we analyzed the differential expression (DE) of transcripts in EVs from cancer patients versus controls (Fig. 3b, Supplementary Table S1). We found $141 \mathrm{mRNAs}$ with significantly different levels; of them, 107 were upregulated and 34 downregulated in the EVs from cancer patients versus controls. Then, we used a recursive feature elimination (RFE) method to select a gene signature predictive of the origin of the sample; a cancer patient or a control sample. The transcripts included in the final signature were BCL10,CXCL11, CYBB and GBP1 and, based on their expression levels, our algorithm was able to classify plasma samples into cancer and control with a receiver operating characteristic (ROC) area under the curve (AUC) of 0.92 to 0.95 (Fig. 3c). The classifier also calculated signature scores for each sample, which were found to be significantly different between cancer patients and controls (Mann-Whitney $\mathrm{U}, p<0.01$; Fig. 3d).

Improvement of classifier performance through removal of genomic DNA. The TRI reagentbased RNA isolation incorporated in our workflow for EV-RNA purification may also co-extract EV-genomic DNA, which could bind to the nCounter probes during hybridization. In consequence, some of the counts detected can correspond to genomic DNA instead of mRNA transcripts, particularly considering that we used a 10 -cycle pre-amplification step. To test if this was the case, we analyzed the effect of adding a DNase treatment step after EV-RNA extraction in the $28 \mathrm{eV}$ samples with remaining material. A dramatic reduction in the number of transcripts detected and the total amount of counts was observed in the EV-RNA samples after DNase treatment (Fig. 4a, Mann-Whitney U, $p<0.01$ in both cases). The average number of transcripts in the 28 sam- 
A

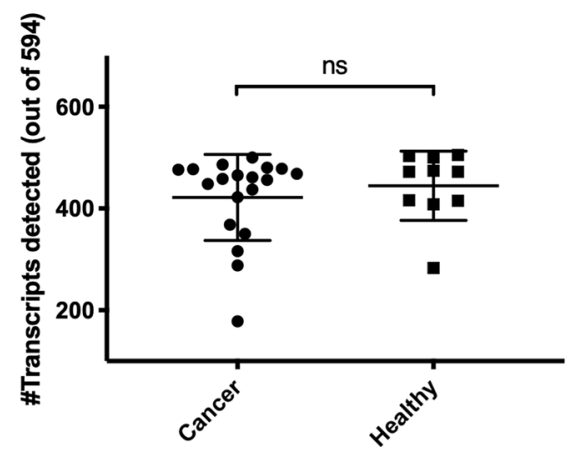

C

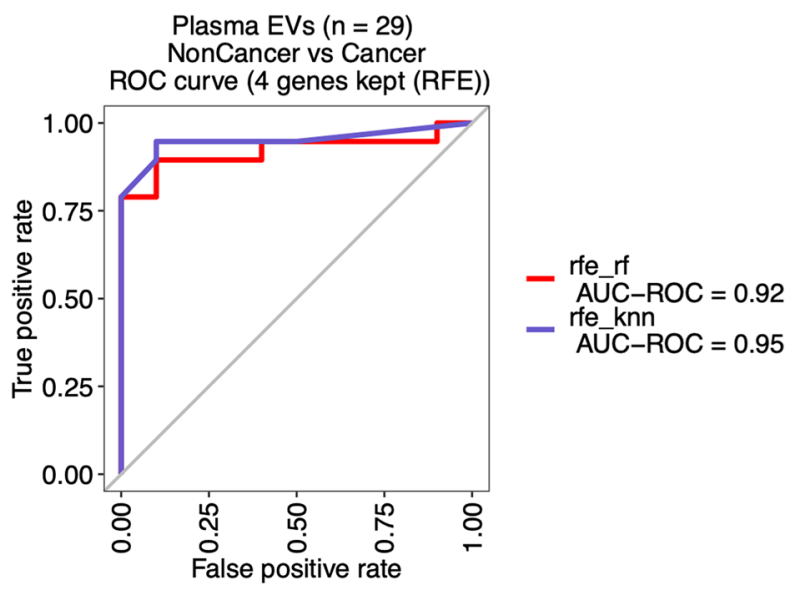

B
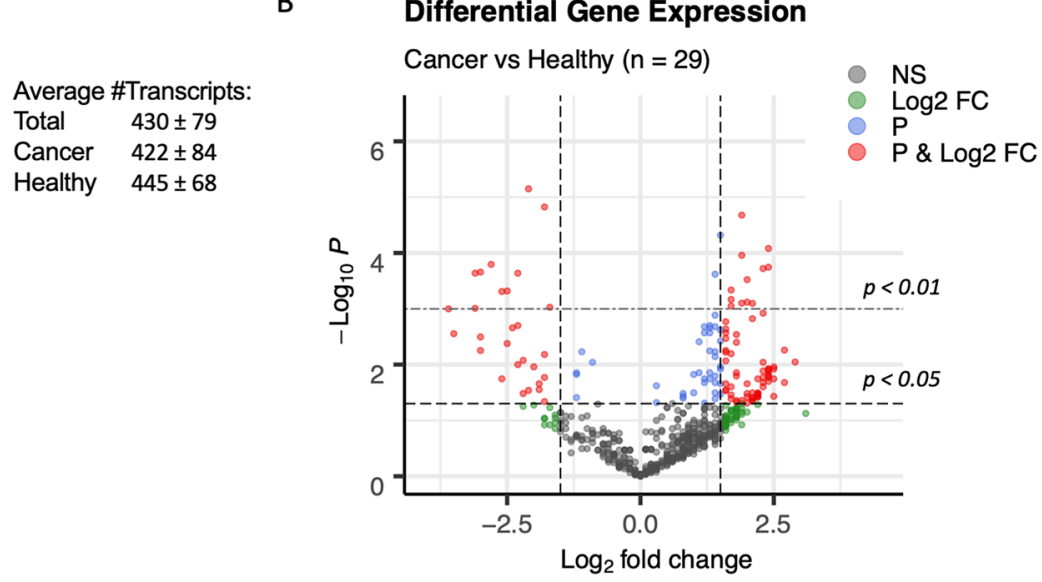

D

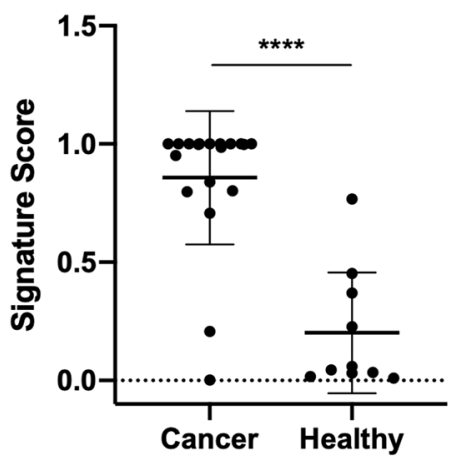

Figure 3. EV-mRNA transcript detection, differential expression analysis and development of a classifier algorithm. (A) Number of transcripts detected in EVs from cancer patients and healthy controls using the Human Immunology v2 nCounter panel, which targets 594 genes (two-tailed Mann-Whitney U test, $p=0.463$ ). (B) Differential expression analysis of log2-normalized counts between cancer patients and healthy controls. The full list of genes differentially expressed is presented in Supplementary Table S1. (C) Area under the ROC curve of the four-gene signature, selected using recursive feature elimination (RFE), to differentiate cancer from control samples. (D) Scores of cancer versus control samples based on expression of the four-gene signature $(p<0.001$ in a two-tailed Mann-Whitney U test). NS, not significant; EVs, extracellular vesicles; ROC, receiver operating characteristic; AUC, area under the curve; RFE, recursive feature elimination; rf, random forest; knn, k-nearest neighbors.

ples decreased from $430 \pm 79$ to $115 \pm 66$ and the total counts dropped more than $90 \%$. As previously observed in samples without DNase treatment, the number of transcripts detected in cancer versus control samples were not significantly different ( $122 \pm 77$ vs $103 \pm 39$, Mann-Whitney's U $p=0.92$, Fig. 4b). In addition, there were no significant differences in the number of transcripts detected between the different tumor types; which included sarcoma, lung, rectal, anal and gastric cancer (Supplementary Fig. S4).

Based on these results, we decided to incorporate a DNase step in our EV-mRNA purification and analysis workflow when working with this panel (Fig. 5), and we confirmed that $500 \mu \mathrm{L}$ still yielded the highest number of transcripts and total counts (Fig. 4c). To further validate the DNase step, we analyzed GAPDH, CCL5 and Caspase 8 (CASP8) expression by qRT-PCR in the $22 \mathrm{eV}$ samples with remaining RNA. We observed a statistically significant correlation between GAPDH and CCL5 expression as measured by nCounter and qRT-PCR (Spearman's $r=0.545, p<0.01$ and $r=0.850, p<0.01$, respectively; Fig. $4 \mathrm{~d}$ ); while CASP 8 transcripts were undetectable in DNase treated EV-RNA by both techniques.

Next, we proceeded to redesign our gene signature and classifier algorithm using the expression data derived from DNase treated EV-mRNAs. We observed that the number of differentially expressed genes in cancer versus control samples dropped from 141 to 43 (Fig. 6a and Supplementary Table S2). Of them, 17 genes (40\%) overlapped with those obtained using non-DNase treated samples (Fig. 6b). Then, we used the RFE algorithm to create a second signature, which included eight genes: CCL5, S100A9, B2M, HLA-B, IL7R, ICAM3, ARHGDIB and PYCARD (Fig. 6a and Supplementary Table S3). The algorithm based on this signature was able to classify the samples into cancer and control with a ROC AUC of 0.99 to 1.00 (Fig. 6c). Also, the signature scores for each sample were found to be significantly different between cancer patients and controls (Mann-Whitney U, $p<0.01$; Fig. 6d). 
A
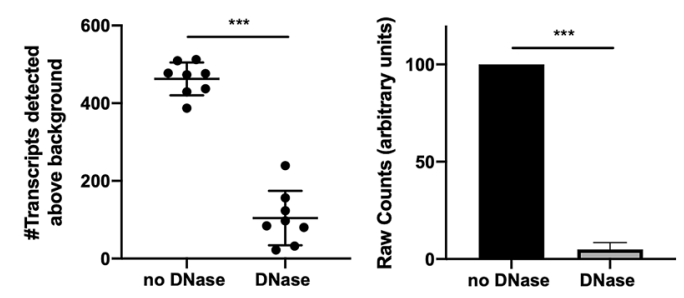

C

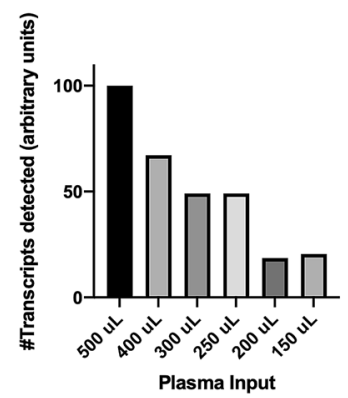

B

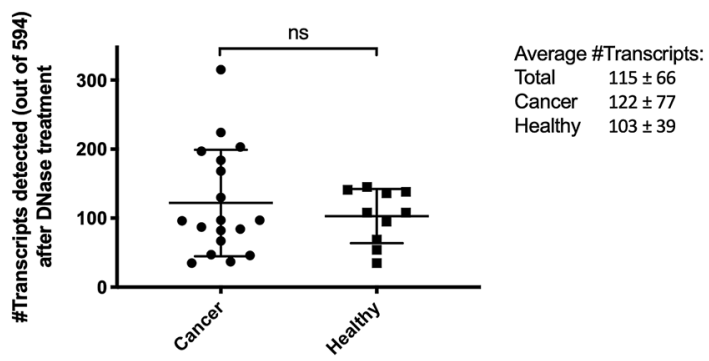

D

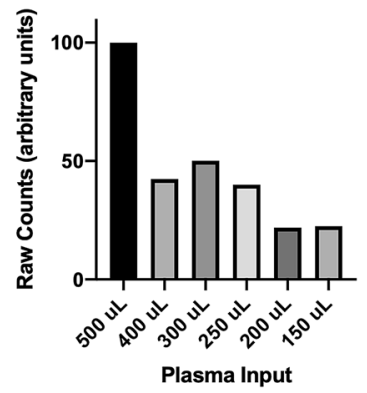

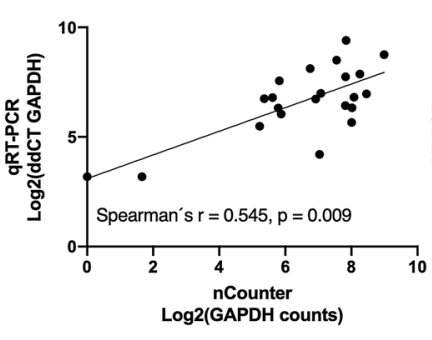

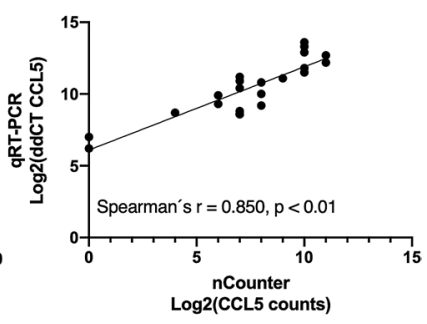

Figure 4. Effect of DNase treatment on the number of transcripts detected, total counts, and additional validation experiments. (A) Number of transcripts detected (left) and total nCounter counts (right) in nonDNase vs. DNase treated EV samples ( $p<0.001$ in a two-tailed Mann-Whitney U test, in both cases). (B) Number of transcripts detected in EVs from cancer patients and control samples using the Human Immunology v2 nCounter panel, after DNase treatment ( $p=0.916$ in a two-tailed Mann-Whitney U test). (C) Effect of input plasma volume (150-500 $\mu \mathrm{L})$ on the final number of transcripts detected (left) or total nCounter counts (right), after DNase treatment. Results were normalized to the counts corresponding to $500 \mu \mathrm{L}$ plasma. (D) Comparison of $\log 2$ counts by nCounter versus ddCT values by qRT-PCR for GAPDH and CCL5 in 22 samples. Spearman's correlation coefficient is indicated. NS, not significant.

The significant decrease in counts after DNase treatment prompted us to further investigate the binding of nCounter probes to genomic DNA co-purified with EV-mRNA. The nCounter Human Immunology V2 Panel targets 594 gene transcripts. The probes for some genes are designed within the same exon ("non-intron spanning"); while other probes ("intron spanning") target sequences corresponding to contiguous exons of the cDNA/ mRNA. Genomic DNA should only bind "non-intron spanning” probes, and we performed an additional experiment to confirm this point. We analyzed an EV sample in quadruplicate, skipping the retrotranscriptase and/or the DNase treatment (Supplementary Table S4). Without DNase treatment, we observed counts for "non-intron spanning" probes (such as CASP8, TNFRSF8 and B2M) both in presence and in absence of the retrotranscriptase step. In contrast, counts for "intron spanning" probes (such as PRKCD, TNFRSF10C, FCER1G) were apparent only if retrotranscription was performed. Finally, DNase treatment induced a significant drop in the counts of "non-intron spanning" but, unexpectedly, also "intron spanning” probes.

\section{Discussion}

The molecules found within EVs, such as mRNAs, are often involved in intercellular communication and represent a potential source for biomarker discovery. However, lack of standardized methods and clinical validation prevents the implementation of EV-derived testing in daily practice. The nCounter platform, which allows for multiplex detection of hundreds of transcripts, has been extensively used in translational research for transcriptomic tumor characterization. In addition, the nCounter Prosigna assay, based on a 50-gene expression signature, has been fully standardized and validated at the clinical level; and received FDA approval in 2013 to predict risk of recurrence in breast cancer ${ }^{15,16,28}$. However, studies investigating the performance of nCounter for mRNA analysis in liquid biopsies are scarce, particularly in the case of EVs.

Here, we present a workflow for nCounter-based analysis of plasma-derived EVs and we demonstrate that it can be used to efficiently detect transcripts in EV-enriched preparations from cancer patients and control samples. The first step of the workflow is plasma processing using the precipitation-based miRCURY kit which, as previously described ${ }^{29,30}$, yielded pellets enriched in EVs. Clinical laboratories do not usually have access to ultracentrifugation and precipitation kits offer several advantages, such as short turn-around time and limited technical requirements. Although $500 \mu \mathrm{L}$ plasma was found to give the highest amount of detected transcripts and total counts by nCounter, lower plasma inputs also yielded valid results. The second step is manual RNA isolation using TRI reagent, which was found to outperform manual purification by TRIzol LS and automated extraction.

The extraction methods tested, including the TRI reagent based manual extraction, did not yield sufficient RNA for direct analysis by nCounter. In consequence, after some optimization experiments, a 10-cycle pre-amplification step was added to our workflow and found to be highly reproducible. Most RNA extraction methods are 


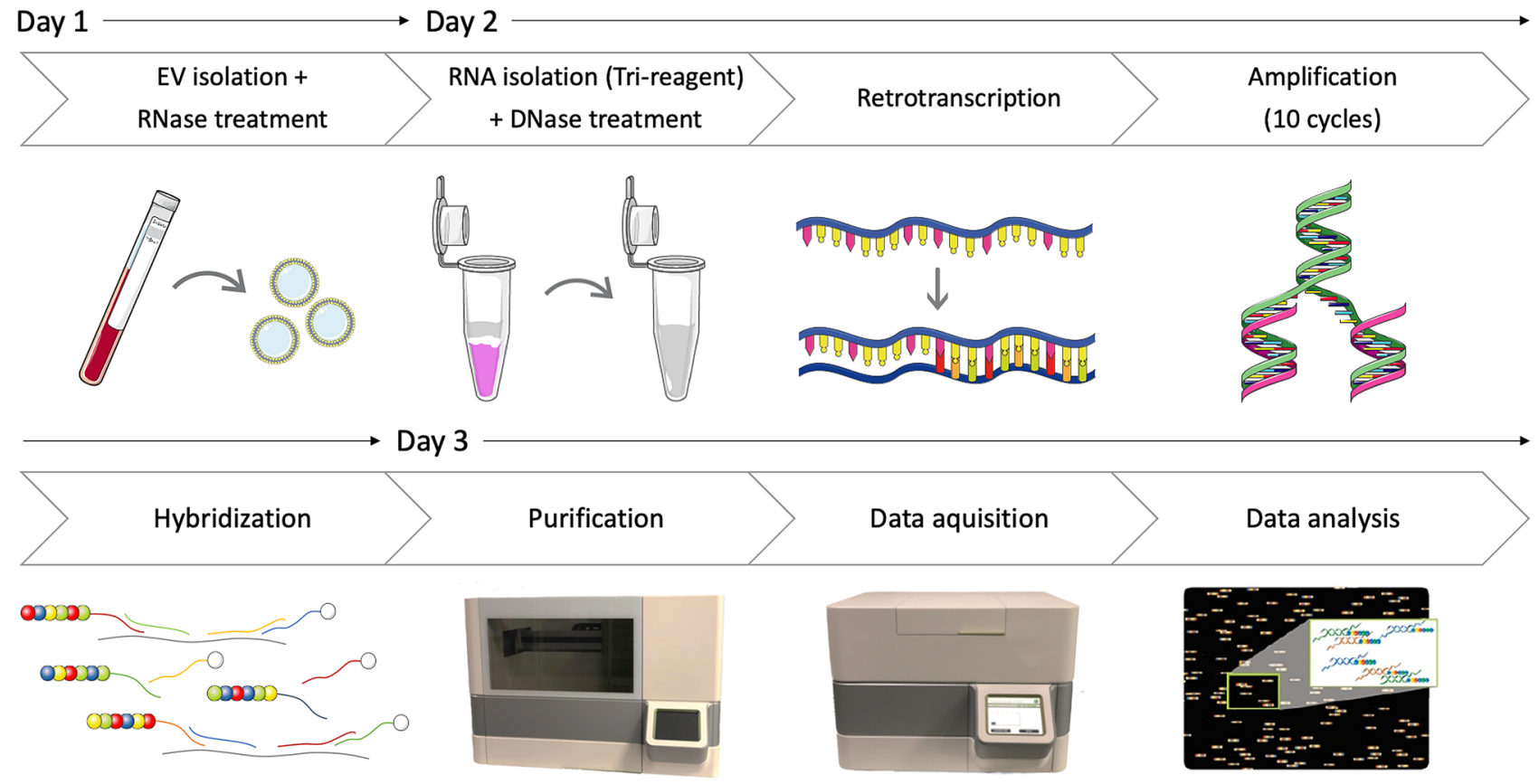

*Only when probes are not designed in an intron-spanning manner

Figure 5. Final workflow for EV-RNA extraction and analysis on the nCounter platform. The miRCURY kit was used to enrich EVs from $500 \mu \mathrm{L}$ plasma and EV-enriched preparations were treated with RNase to remove extra-vesicular RNA. Then, EV-RNA was extracted using TRI reagent and treated with DNase to remove genomic DNA. DNase treatment is only necessary when probes are not designed in an intron-spanning manner. Next, we performed retrotranscription and a 10-cycle pre-amplification, followed by hybridization, purification on the nCounter prep-station and analysis on the nCounter digital analyzer. EVs, extracellular vesicles. Part of this figure was modified from SMART (Servier Medical Art), licensed under a Creative Common Attribution 3.0 Generic License. http://smart.servier.com/.

known to co-purify genomic DNA (gDNA), and this was also our case. The EV-associated gDNA could be located in the interior of the vesicles or be attached to the membranous surface, and further research is needed to clarify this issue. More importantly, it has been described that simultaneous isolation of EV-derived genomic DNA during EV-RNA extraction can affect the amount of detected transcripts ${ }^{31}$. In consequence, we tested the effect of adding a DNase treatment step to our methodology and found a significant decrease in total counts and number of transcripts detected. Interestingly, while the expression of many genes became undetectable after DNase treatment, the counts for some transcripts were maintained. These observations suggested that co-extracted EVgenomic DNA was amplified during the pre-amplification step and could hybridize to the "non-intron spanning" probes in the nCounter panel. In contrast, probes designed in an "intron-spanning" manner should not hybridize to EV-DNA. Validation experiments confirmed this point (Supplementary Table S4). Unexpectedly, we observed a sharp decrease also in the counts of "intron-spanning" probes after DNase treatment, strongly suggestive of EV-RNA degradation or, more likely, EV-RNA loss during the purification steps needed to remove the DNase. In consequence, appropriate design of probes should be preferred over DNase treatment when analyzing samples with low amounts of RNA that require pre-amplification. Since we were using a pre-designed panel that could not be modified, we added a DNase treatment step to our final protocol for EV-mRNA analysis (Fig. 5). As an additional validation of the entire workflow, we re-analyzed 22 DNase treated samples for GAPDH, CASP8 and CCL5 expression by qRT-PCR. Similarly to previous reports ${ }^{22,32}$, we found a statistically significant correlation between the expression levels obtained by qRT-PCR and nCounter.

Next, we investigated if our workflow for EV-mRNA analysis could be used to develop gene signatures. To this end, we performed differential expression analysis of the nCounter results obtained for control and cancer samples. The signature-based algorithm obtained for EV-mRNA (DNase treated) showed an improved classifier performance in comparison with EV-nucleic acids (non-DNase treated); with ROC-AUCs of 0.99-1.00 versus $0.92-0.95$ for the discrimination of control versus cancer, respectively. This result is coincident with a previous report where a DNA removal step was shown to reduce signature noise during algorithm development and yielded better classification results ${ }^{33}$. The genes selected for our EV-mRNA expression signature are CCL5, S100A9, B2M, HLA-B, IL7R, ICAM3, ARHGDIB and PYCARD; which are involved in several immune-related pathways such as cytokine signaling, innate immune system or lymphocyte activation (Fig. 6a and Supplementary Table S3). CCL5, B2M, HLA-B and IL7R are all related to cytokine signaling and lymphocyte activation, and are thus known as immunomodulators. Interestingly, these four transcripts were downregulated in cancer samples, suggesting differences in immune system activation through cytokine induction between cancer patients and controls. PYCARD is also proposed to be involved in lymphocyte activation and was found to be upregulated in 
A

Differential Gene Expression

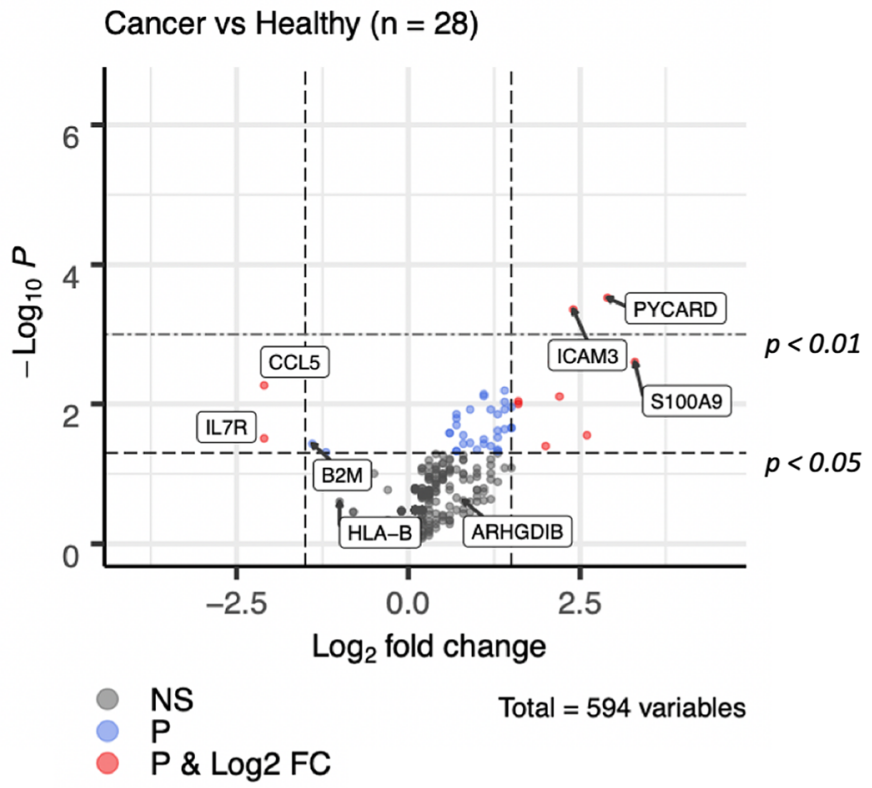

C

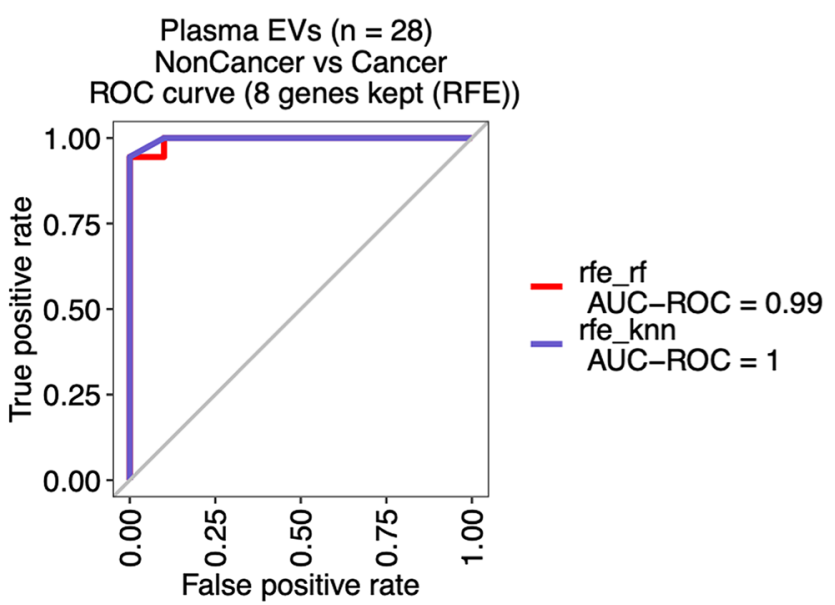

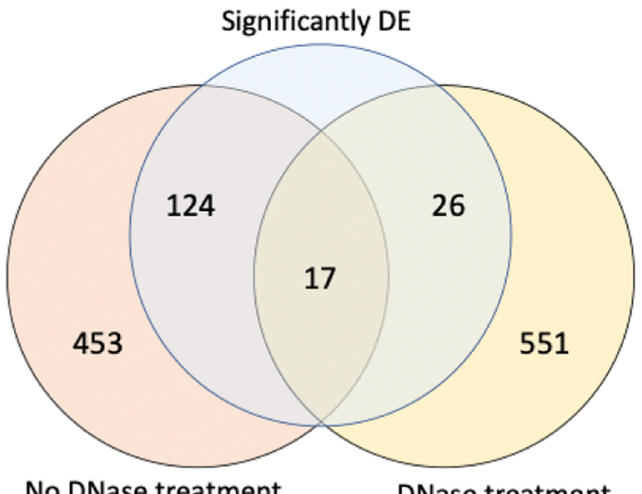

No DNase treatment
D

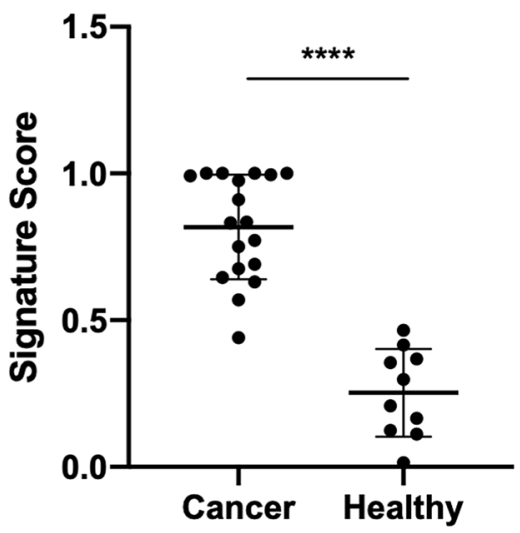

Figure 6. Effect of DNase treatment on differential expression analysis and classifier development. (A) Differential expression analysis of log2-normalized counts after DNase treatment between cancer patients and control samples. The full list of genes differentially expressed is presented in Supplementary Table S2. Labels indicate the eight transcripts selected for the final classification signature. (B) Out of 594 genes in the panel, 17 showed differential expression independently of DNase treatment. (C) Recursive feature elimination (RFE) was used to select an eight-gene signature that could distinguish between samples derived from cancer patients and controls. (D) Scores of cancer versus control samples based on expression of the eight gene signature $(\mathrm{p}<0.001$ in a two-tailed Mann-Whitney U test). DE, differentially expressed; EVs, extracellular vesicles; ROC, receiver operating characteristic; AUC, area under the curve; RFE, recursive feature elimination; rf, random forest; knn, k-nearest neighbors.

cancer patients. A previous study found that PYCARD could suppress apoptosis of cancer cells in gastric cancer ${ }^{34}$, potentially explaining the observed higher expression in EVs from cancer patients.

Two studies have used nCounter for analysis of mRNA isolated from blood. Kossenkov et $\mathrm{al}^{23}$ developed a pulmonary node classifier to differentiate malignant from non-malignant nodules previously detected by lowdose CT. Since the authors made use of whole blood, the quantities of purified RNA were significantly higher than those in our EV-based study ( $3 \mu \mathrm{g} / 2.5 \mathrm{~mL}$ blood versus $0.01 \mu \mathrm{g} / 2.5 \mathrm{~mL}$, respectively), avoiding the need for a pre-amplification step. Beck et $\mathrm{a}^{21}$ used a combination of CTC-RNA and cfRNA to profile tumor-associated biomarkers and correlate them with diagnostics and survival. The quantities of RNA were similar to those obtained in our study and a pre-amplification step was also added. 
Our study shows several limitations. First, although purification of EVs from plasma yielded pellets that were found to be enriched in EVs, such precipitation techniques can also isolate a significant fraction of proteins and lipoproteins ${ }^{35,36}$. Second, there are no validated housekeeping genes for normalization of EV-mRNAs and we had to use the total amount of counts, as described ${ }^{37-39}$. Third, although nCounter presents many advantages, it has also a few limitations when compared to other multiplex techniques such as RNAseq. For instance, since nCounter works with gene panels, no new transcripts can be found. Finally, our aim was to establish a workflow for the nCounter analysis of EV-mRNA and the mixed patient population we used to demonstrate the validity of our approach was not the most appropriate to develop a clinically useful signature. Much larger patient cohorts would be needed to train and validate signatures that could differentiate between cancer patients and controls.

In summary, to the best of our knowledge, this proof-of-concept study is the first to demonstrate that the nCounter platform can be used to reproducibly detect plasma EV-mRNA transcripts. Differential expression analysis can then be implemented for biomarker assay development. Our work paves the way for widespread testing of EV-mRNA expression in blood and other fluids, and subsequent selection of signatures useful in the clinical setting.

\section{Methods}

Patient samples. This study was carried out in accordance with the principles of the Declaration of Helsinki under an approved protocol of the institutional review board of Quirón Hospitals. We selected for the study all blood samples from advanced stage cancer patients arriving to our institution in a two month period with sufficient volume for EV extraction after routine genetic testing $(n=19)$. Blood samples from 10 healthy controls were also collected (Table 1). Written informed consent was obtained from all participants and documented; samples were de-identified for confidentiality. Clinical information collected from each participant was limited to gender, age, tumor type and stage.

Extracellular vesicle enrichment. Whole blood samples $(10 \mathrm{~mL})$ were collected in sterile EDTA Vacutainer tubes (BD, Plymouth, UK) and centrifugated twice at $1000 \times \mathrm{g}$ for $10 \mathrm{~min}$ at room temperature (RT). Plasma samples were stored at $-80{ }^{\circ} \mathrm{C}$ until further processing. The miRCURY Exosome Serum/Plasma Kit (Qiagen, Hilden, Germany) was used to enrich for EVs from $500 \mu \mathrm{L}$ plasma, according to the manufacturer's instructions, unless otherwise specified. In short, dead cells and debris (including platelets and fibrin) were cleared with thrombin and centrifugation. Precipitation Buffer was added, samples were incubated overnight at $4{ }^{\circ} \mathrm{C}$ and the EV fraction was pelleted by centrifugation. Supernatants were collected and stored for separate analysis. EV enriched pellets were resuspended for further processing.

EV characterization by western blot. EV enriched pellets were resuspended in $300 \mu \mathrm{L}$ ice-cold radioimmunoprecipitation assay buffer containing protease inhibitor mixture (Roche Applied Science, Penzberg, Germany), as previously described ${ }^{40}$. Samples were incubated on ice for $30 \mathrm{~min}$, homogenized and centrifugated $15 \mathrm{~min}, 12.000 \times \mathrm{g}$ at $4{ }^{\circ} \mathrm{C}$. Supernatants (lysates) were collected and $80 \mu \mathrm{g}$ proteins were electrophoresed on $10 \%$ SDS-polyacrylamide gels (Life Technologies, Carlsbad, CA, USA) and transferred to PVDF membranes (Bio-Rad Laboratories Inc., Hercules, CA, USA). Membranes were blocked in Odyssey Blocking Buffer (Li-Cor Biosciences, Lincoln, NE, USA). All target proteins were immunoblotted with appropriate primary and horseradish peroxidase (HRP)-conjugated secondary antibodies (Supplementary Table S5). Chemiluminescent bands were detected in a ChemiDoc MP Imaging System (Bio-Rad Laboratories Inc.).

EV characterization with cryogenic electron microscopy. Cryogenic Electron Microscopy (CryoEM) was performed by the Microscopy Service of the Universitat Autonoma de Barcelona (UAB), and used for direct visualization of EVs using the TEM JEOL $2011200 \mathrm{kV}$. As previously described ${ }^{41}, 2 \mu \mathrm{L}$ volume of the resuspended EV sample was added to a carbon TEM grid. The grid was transferred onto the cyro-preparation chamber of a Leica electron microscope, containing a liquid ethane bath cooled to $-180^{\circ} \mathrm{C}$. Using a piece of filter paper, the EV solution was taken off the grid and plunged into the liquid ethane. The orifice trapped frozen EV solution was assembled into a plunger (Leica EM GP) and blotted with Whatman No. 1 filter paper. The grid was placed in a liquid nitrogen bath, and then loaded into a liquid nitrogen-cooled TEM grid holder. The grid holder was placed into a JEOL 2011 TEM microscope. Imaging was performed using a Gatan UltraScan US1000 CCD camera and data was analyzed with Digital Micrograph 1.8

RNA extraction. EV-enriched pellets were treated with $4 \mu \mathrm{g} / \mathrm{mL}$ RNase A from bovine pancreas (SigmaAldrich, St. Louis, MO) for $1 \mathrm{~h}$ at $37^{\circ} \mathrm{C}$, to remove extra-vesicular RNA not associated to EVs. For TRIzol LS Reagent (Thermo Fisher Scientific, Waltham, MA) and TRI Reagent (MRC, Cincinnati, OH) extraction, TRIzol solutions were added to a final volume of $1 \mathrm{~mL}$ and incubated at RT for 20 min to inactivate RNase A and lyse the EVs. Then, $200 \mu \mathrm{L}$ Chloroform: Isoamyl Alcohol (24:1) (Panreac Química SLU, Barcelona, Spain) was added and samples were vigorously vortexed and centrifuged at $12,000 \times \mathrm{g}$ for $15 \mathrm{~min}$ at $4{ }^{\circ} \mathrm{C}$. The aqueous upper layer was kept and RNA was precipitated adding $2.5 \mu \mathrm{L}$ Glycogen (Merck KGaA, Darmstadt, Germany) and $500 \mu \mathrm{L}$ 2-propanol (Merck KGaA), incubating $10 \mathrm{~min}$ at RT and centrifugating for $10 \mathrm{~min}, 12,000 \times \mathrm{g}$, at $4{ }^{\circ} \mathrm{C}$. The final RNA pellet was washed with 75\% ethanol, air dried and dissolved in $20 \mu \mathrm{L}$ nuclease free water. The QIAsymphony DSP Virus/Pathogen Kit was also tested in the automated QIAsymphony SP System (Qiagen) for RNA extraction from EVs, according to the manufacturer's instructions. 
Electrophoretic analysis of RNA. The approximate quantity and size distribution of the isolated EVRNA was evaluated using the Bioanalyzer RNA 6000 Pico Assay (Agilent Technologies, Santa Clara, CA) according to manufacturer instructions.

DNase treatment. In order to remove co-isolated DNA, the EV-RNA samples were treated with the DNAfree DNA Removal Kit (Thermo Fisher Scientific), according to manufacturer instructions. In short, $1 \mu \mathrm{L}$ DNase buffer and $0.5 \mu \mathrm{L}$ enzyme were added to $7.5 \mu \mathrm{L}$ RNA sample, followed by incubation at $37^{\circ} \mathrm{C}$ for $30 \mathrm{~min}$ and DNase removal.

Gene expression analysis using nCounter. The nCounter Low RNA Input Amplification Kit (NanoString Technologies, Seattle, WA) was used to retrotranscribe and pre-amplify $4 \mu \mathrm{L}$ EV-derived RNA using 10 cycles. Retrotranscription was carried out in $0.5 \mathrm{~mL}$ tubes while pre-amplification, using primers targeting the genes of the Human Immunology V2 Panel (NanoString Technologies), was performed in 384-well plates to prevent sample evaporation. In parallel, a Moloney Murine Leukema Virus (M-MLV) Reverse Transcriptase Enzyme (Thermo Fisher Scientific) was also tested for cDNA synthesis. The Human Immunology V2 Panel (NanoString Technologies) was used to analyze EV-derived, pre-amplified cDNA according to manufacturer instructions. This panel targets 594 general genes involved in the immune response such as cytokines, enzymes, interferons and their receptors. Samples were hybridized for $18 \mathrm{~h}$ at $65^{\circ} \mathrm{C}$.

Data normalization and analysis. Raw nCounter counts of expressed genes were normalized in R and $\mathrm{R}$ studio (version 3.6.3, https://cran.r-project.org/bin/macosx/) using the R package NanoStringNorm ${ }^{42}$. Normalization was performed following several steps: technical assay variability normalization using the geometric mean of the positive control probes, background correction using the mean plus two times standard deviation (SD) of the negative control probes, and sample content normalization using the total amount of counts for each sample. Normalized counts were log2-transformed, and used for differential expression (DE) analysis. Log2 fold change (FC) of each gene was calculated as the ratio of average log- 2 transformed counts of the cancer patient cohort versus the control cohort. Volcano plots were used to visualize log2 FC on the $\mathrm{x}$-axis and nominal p-values on the y-axis. GraphPad Prism software (version 9.0.0; https://www.graphpad.com/scientific-software/ prism/) was used for other statistical testing and to create figures.

Classifier algorithm development. Optimal gene selection was performed using recursive feature elimination (RFE). To this end, a leave-one-out cross validation (LOOCV) algorithm was used on the full Human Immunology V2 gene Panel. The number of genes to select was set at 4, 8, 16 or 579 and the amount of genes that yielded optimal performance after cross-validation was automatically selected. Classification was performed with the selected gene signature using random forest (RF) and k-nearest neighbors (KNN) classifiers with three iterations. The model with the highest accuracy was then selected as the final model. Signature scores for each sample were derived from the final model.

Gene expression analysis using qRT-PCR. Complementary DNA (cDNA) was synthesized using the M-MLV Reverse Transcriptase Enzyme (Thermo Fisher Scientific). Hereafter, cDNA was added to TaqMan Universal Master Mix (Applied Biosystems) in a $12.5 \mu \mathrm{L}$ reaction with specific primers and probe designed for each gene. The primer and probe sets were designed using Primer Express Software (version 3.0.1; https://www.therm ofisher.com/order/catalog/product/4363993\#/4363993) according to their Ref Seq (http://www.ncbi.nlm.nih. gov/LocusLink) Gene-specific primers were designed as follows: GAPDH, forward: 5'-TGACCTCAACTACAT GGTTTACATGTT-3' and reverse: 5'-TGACGGTGCCATGGAATTT-3'; Caspase 8, forward: 5'-CAGGGCTCA AATTTCTGCCTAC-3' and reverse: 5'-GAAGAAGTGAGCAGATCAGAATTGAG-3'; CCL5, forward: 5' CAT CTGCCTCCCCATATTCCT 3' and reverse: 5' AGTGGGCGGGCAATGTAG 3'. Quantification of gene expression was performed using the QuantStudio 7 Flex Real-Time PCR System (Thermo Fisher Scientific). Expression levels of mRNA were expressed as arbitrary units based on Ct values. Commercial RNAs were used as controls (liver and lung; Stratagene, La Jolla, CA). In all quantitative experiments, a sample was considered not evaluable when the standard deviation of the Ct values was $>0.30$ in 2 independent analyses.

\section{Data availability}

Supplementary information is available for this paper.

Received: 22 October 2020; Accepted: 29 January 2021

Published online: 12 February 2021

\section{References}

1. Bray, F. et al. Global cancer statistics 2018: GLOBOCAN estimates of incidence and mortality worldwide for 36 cancers in 185 countries. CA Cancer J. Clin. 68, 394-424. https://doi.org/10.3322/caac.21492 (2018).

2. Margolis, L. \& Sadovsky, Y. The biology of extracellular vesicles: the known unknowns. PLoS Biol. 17, e3000363. https://doi. org/10.1371/journal.pbio.3000363 (2019).

3. Reclusa, P. et al. Exosomes genetic cargo in lung cancer: a truly Pandora's box. Transl. Lung Cancer Res. 5, 483-491. https://doi. org/10.21037/tlcr.2016.10.06 (2016).

4. Jeppesen, D. K. et al. Reassessment of exosome composition. Cell 177, 428-445 e418. https://doi.org/10.1016/j.cell.2019.02.029 (2019).

5. Fujita, Y., Yoshioka, Y. \& Ochiya, T. Extracellular vesicle transfer of cancer pathogenic components. Cancer Sci. 107, 385-390. https ://doi.org/10.1111/cas.12896 (2016). 
6. Kalluri, R. The biology and function of exosomes in cancer. J. Clin. Invest. 126, 1208-1215. https://doi.org/10.1172/JCI81135 (2016).

7. Nolte-'t Hoen, E. N. et al. Deep sequencing of RNA from immune cell-derived vesicles uncovers the selective incorporation of small non-coding RNA biotypes with potential regulatory functions. Nucleic Acids Res. 40, 9272-9285. https://doi.org/10.1093/ nar/gks658 (2012).

8. Stevic, I., Buescher, G. \& Ricklefs, F. L. Monitoring therapy efficiency in cancer through extracellular vesicles. Cells https://doi. org/10.3390/cells $9010130(2020)$.

9. Reclusa, P. et al. Exosomes as diagnostic and predictive biomarkers in lung cancer. J. Thorac. Dis. 9, S1373-S1382. https://doi. org/10.21037/jtd.2017.10.67 (2017).

10. Narrandes, S. \& Xu, W. Gene expression detection assay for cancer clinical use. J. Cancer 9, 2249-2265. https://doi.org/10.7150/ jca.24744 (2018).

11. Segundo-Val, I. S. \& Sanz-Lozano, C. S. Introduction to the gene expression analysis. Methods Mol. Biol. 1434, 29-43. https://doi. org/10.1007/978-1-4939-3652-6_3(2016).

12. Prokopec, S. D. et al. Systematic evaluation of medium-throughput mRNA abundance platforms. RNA 19, 51-62. https://doi. org/10.1261/rna.034710.112 (2013).

13. Geiss, G. K. et al. Direct multiplexed measurement of gene expression with color-coded probe pairs. Nat. Biotechnol. 26, 317-325. https://doi.org/10.1038/nbt1385 (2008).

14. Warren, S. Simultaneous, multiplexed detection of RNA and protein on the nanostring((R)) nCounter((R)) platform. Methods Mol. Biol. 1783, 105-120. https://doi.org/10.1007/978-1-4939-7834-2_5 (2018).

15. Gnant, M. et al. Predicting distant recurrence in receptor-positive breast cancer patients with limited clinicopathological risk: using the PAM50 risk of recurrence score in 1478 postmenopausal patients of the ABCSG-8 trial treated with adjuvant endocrine therapy alone. Ann. Oncol. 25, 339-345. https://doi.org/10.1093/annonc/mdt494 (2014).

16. Hannouf, M. B. et al. Cost-effectiveness analysis of multigene expression profiling assays to guide adjuvant therapy decisions in women with invasive early-stage breast cancer. Pharmacogenomics J. 20, 27-46. https://doi.org/10.1038/s41397-019-0089-x (2020).

17. Ayers, M. et al. IFN-gamma-related mRNA profile predicts clinical response to PD-1 blockade. J. Clin. Invest. 127, 2930-2940. https://doi.org/10.1172/JCI91190 (2017).

18. Giménez-Capitán, A. et al. Multiplex detection of clinically relevant mutations in liquid biopsies of cancer patients using the nCounter Platform. Clin. Chem. (2020) (in press).

19. Kamyabi, N. et al. Isolation and mutational assessment of pancreatic cancer extracellular vesicles using a microfluidic platform. Biomed. Microdev. 22, 23. https://doi.org/10.1007/s10544-020-00483-7 (2020).

20. Porras, T. B., Kaur, P., Ring, A., Schechter, N. \& Lang, J. E. Challenges in using liquid biopsies for gene expression profiling. Oncotarget 9, 7036-7053. https://doi.org/10.18632/oncotarget.24140 (2018).

21. Beck, T. N. et al. Circulating tumor cell and cell-free RNA capture and expression analysis identify platelet-associated genes in metastatic lung cancer. BMC Cancer 19, 603. https://doi.org/10.1186/s12885-019-5795-x (2019).

22. Wu, T. C. et al. IL1 receptor antagonist controls transcriptional signature of inflammation in patients with metastatic breast cancer. Cancer Res. 78, 5243-5258. https://doi.org/10.1158/0008-5472.CAN-18-0413 (2018).

23. Kossenkov, A. V. et al. A gene expression classifier from whole blood distinguishes benign from malignant lung nodules detected by low-dose CT. Cancer Res. 79, 263-273. https://doi.org/10.1158/0008-5472.CAN-18-2032 (2019).

24. Garcia-Contreras, M. et al. Plasma-derived exosome characterization reveals a distinct microRNA signature in long duration Type 1 diabetes. Sci. Rep. 7, 5998. https://doi.org/10.1038/s41598-017-05787-y (2017).

25. Vicentini, C. et al. Exosomal miRNA signatures of pancreatic lesions. BMC Gastroenterol. 20, 137. https://doi.org/10.1186/s1287 6-020-01287-y (2020).

26. Emelyanov, A. et al. Cryo-electron microscopy of extracellular vesicles from cerebrospinal fluid. PLoS ONE 15, e0227949. https:// doi.org/10.1371/journal.pone.0227949 (2020).

27. Rasch, M. R. et al. Hydrophobic gold nanoparticle self-assembly with phosphatidylcholine lipid: membrane-loaded and Janus vesicles. Nano Lett. 10, 3733-3739. https://doi.org/10.1021/nl102387n (2010).

28. Dowsett, M. et al. Comparison of PAM50 risk of recurrence score with oncotype DX and IHC4 for predicting risk of distant recurrence after endocrine therapy. J. Clin. Oncol. 31, 2783-2790. https://doi.org/10.1200/JCO.2012.46.1558 (2013).

29. Pasquali, L. et al. Circulating microRNAs in extracellular vesicles as potential biomarkers for psoriatic arthritis in patients with psoriasis. J. Eur. Acad. Dermatol. Venereol. 34, 1248-1256. https://doi.org/10.1111/jdv.16203 (2020).

30. Helwa, I. et al. A comparative study of serum exosome isolation using differential ultracentrifugation and three commercial reagents. PLoS ONE 12, e0170628. https://doi.org/10.1371/journal.pone.0170628 (2017).

31. Naderi, A. et al. Expression microarray reproducibility is improved by optimising purification steps in RNA amplification and labelling. BMC Genomics 5, 9. https://doi.org/10.1186/1471-2164-5-9 (2004).

32. Richard, A. C. et al. Comparison of gene expression microarray data with count-based RNA measurements informs microarray interpretation. BMC Genomics 15, 649. https://doi.org/10.1186/1471-2164-15-649 (2014).

33. Esmaeili, M. et al. Noninvasive sexing of human preimplantation embryos using RT-PCR in the spent culture media: a proof-ofconcept study. Eur. J. Obstet. Gynecol. Reprod. Biol. 252, 89-93. https://doi.org/10.1016/j.ejogrb.2020.06.023 (2020).

34. Deswaerte, V. et al. Inflammasome adaptor ASC suppresses apoptosis of gastric cancer cells by an il18-mediated inflammationindependent mechanism. Cancer Res. 78, 1293-1307. https://doi.org/10.1158/0008-5472.CAN-17-1887 (2018).

35. Johnsen, K. B., Gudbergsson, J. M., Andresen, T. L. \& Simonsen, J. B. What is the blood concentration of extracellular vesicles? Implications for the use of extracellular vesicles as blood-borne biomarkers of cancer. Biochim. Biophys. Acta Rev. Cancer 109-116, 2019. https://doi.org/10.1016/j.bbcan.2018.11.006 (1871).

36. Sodar, B. W. et al. Low-density lipoprotein mimics blood plasma-derived exosomes and microvesicles during isolation and detection. Sci. Rep. 6, 24316. https://doi.org/10.1038/srep24316 (2016).

37. Rozowsky, J. et al. exceRpt: a comprehensive analytic platform for extracellular RNA profiling. Cell Syst. 8, 352-357 e353. https:// doi.org/10.1016/j.cels.2019.03.004 (2019).

38. Morhayim, J. et al. Molecular characterization of human osteoblast-derived extracellular vesicle mRNA using next-generation sequencing. Biochim. Biophys. Acta Mol. Cell Res. 1133-1141, 2017. https://doi.org/10.1016/j.bbamcr.2017.03.011 (1864).

39. Koppers-Lalic, D. et al. Noninvasive prostate cancer detection by measuring miRNA variants (isomiRs) in urine extracellular vesicles. Oncotarget 7, 22566-22578. https://doi.org/10.18632/oncotarget.8124 (2016).

40. Bracht, J. W. P. et al. Osimertinib and pterostilbene in EGFR-mutation-positive non-small cell lung cancer (NSCLC). Int. J. Biol. Sci. 15, 2607-2614. https://doi.org/10.7150/ijbs.32889 (2019).

41. Puente-Massaguer, E., Lecina, M. \& Godia, F. Nanoscale characterization coupled to multi-parametric optimization of Hi5 cell transient gene expression. Appl. Microbiol. Biotechnol. 102, 10495-10510. https://doi.org/10.1007/s00253-018-9423-5 (2018).

42. Waggott, D. et al. NanoStringNorm: an extensible R package for the pre-processing of NanoString mRNA and miRNA data. Bioinformatics 28, 1546-1548. https://doi.org/10.1093/bioinformatics/bts188 (2012). 


\title{
Acknowledgements
}

The investigators wish to thank the patients for kindly accepting to donate samples to this study. We also thank all the physicians who collaborated by providing clinical information. Finally, we would like to thank colleagues and collaborators for their constructive feedback on this manuscript.

\section{Author contributions}

J.W.P.B., C.P.V. and M.A.M.V. conceptualized and designed the experiments. J.W.P.B. and A.G.C. performed the experiments. N.P. and J.W.P.B. performed data analysis. J.W.P.B. and M.A.M.V. wrote the main manuscript and prepared the figures. C.Y.H., S.W. and R.R. provided editing, comments, and experimental guidance. All authors reviewed the manuscript.

\section{Funding}

This project has received funding from a European Union's Horizon 2020 research and innovation program under the Marie Skłodowska-Curie Grant Agreement ELBA No. 765492.

\section{Competing interests}

C.Y.H. and S.W. were full-time employees of NanoString Inc. when the project was carried out. The rest of the authors declare no competing interests.

\section{Additional information}

Supplementary Information The online version contains supplementary material available at https://doi. org/10.1038/s41598-021-83132-0.

Correspondence and requests for materials should be addressed to J.W.P.B. or M.A.M.-V.

Reprints and permissions information is available at www.nature.com/reprints.

Publisher's note Springer Nature remains neutral with regard to jurisdictional claims in published maps and institutional affiliations.

\begin{abstract}
Open Access This article is licensed under a Creative Commons Attribution 4.0 International License, which permits use, sharing, adaptation, distribution and reproduction in any medium or format, as long as you give appropriate credit to the original author(s) and the source, provide a link to the Creative Commons licence, and indicate if changes were made. The images or other third party material in this article are included in the article's Creative Commons licence, unless indicated otherwise in a credit line to the material. If material is not included in the article's Creative Commons licence and your intended use is not permitted by statutory regulation or exceeds the permitted use, you will need to obtain permission directly from the copyright holder. To view a copy of this licence, visit http://creativecommons.org/licenses/by/4.0/.
\end{abstract}

(C) The Author(s) 2021 